\title{
Refinement of the logarithmic law of the wall
}

\author{
F. Laadhari* \\ Laboratoire de Mécanique des Fluides et d'Acoustique, \\ Université de Lyon, Université Claude Bernard Lyon 1, \\ Ecole Centrale de Lyon, INSA de Lyon, CNRS UMR 5509, \\ 36 Avenue Guy de Collongue, F-69134 Ecully, France
}

(Received 16 July 2018; published 30 May 2019)

\begin{abstract}
Available direct numerical simulation of turbulent channel flow at moderately high Reynolds numbers data show that the logarithmic diagnostic function is a linearly decreasing function of the outer-normalized wall distance $\eta=y / \delta$ with a slope proportional to the von Kármán constant, $\kappa=0.4$. The validity of this result for turbulent pipe and boundary layer flows is assessed by comparison with the mean velocity profile from experimental data. The results suggest the existence of a flow-independent logarithmic law $\bar{U}^{+}=\bar{U} / u_{\tau}=(1 / \kappa) \ln \left(y^{*} / a\right)$, where $y^{*}=y U_{S} / v$ with $U_{S}=y S(y)$ being the local shear velocity and the two flow-independent constants $\kappa=0.4$ and $a=0.36$. The range of its validity extends from the inner-normalized wall distance $y^{+}=300$ up to half the outer-length scale $\eta=0.5$ for internal flows, and $\eta=0.2$ for zero-pressure-gradient turbulent boundary layers. Likewise, and within the same range, the mean velocity deficit follows a flow-dependent logarithmic law as a function of a local mean-shear-based coordinate. Furthermore, it is illustrated how the classical friction laws for smooth pipe and zero-pressure-gradient turbulent boundary layer are recovered from this scaling.
\end{abstract}

DOI: 10.1103/PhysRevFluids.4.054605

\section{INTRODUCTION}

The Prandtl-von Kármán logarithmic law [1-3] for the mean velocity profile in canonical turbulent wall-bounded flows (plane channel, circular pipe, and zero-pressure-gradient boundary layer) is one of the most important results in the field of turbulence, both because of its obvious practical interest and of the elegance of its derivation. The law of the wall has indeed been obtained either with dimensional analysis, similarity arguments or closure assumptions and is well supported by experimental data, leading to the inner-scaled law of the wall and outer-scaled defect law, respectively:

$$
\begin{aligned}
\bar{U}^{+}\left(y^{+}\right) & =\frac{1}{\kappa} \ln y^{+}+A, \\
\bar{U}_{c}^{+}\left(\delta^{+}\right)-\bar{U}^{+}\left(y^{+}\right) & =-\frac{1}{\kappa} \ln \eta+B,
\end{aligned}
$$

where $\bar{U}^{+}=\bar{U} / u_{\tau}$ and $y^{+}=y u_{\tau} / \nu . \bar{U}$ is the mean streamwise velocity, $y$ is the distance from the wall, $v$ is the kinematic viscosity, and $u_{\tau}$ is the friction velocity defined from the wall shear stress $\tau_{w}$ as $u_{\tau}=\sqrt{\tau_{w} / \rho}$, where $\rho$ is the fluid density. $\bar{U}_{c}$ is the mean centerline, or outer velocity, and $\eta=y / \delta$, with $\delta$ being the channel half width, the pipe radius, or the boundary layer thickness. The superscript $(+)$ denotes scaling with inner variables, $u_{\tau}$ and $\nu$, and $R_{\tau}=\delta^{+}$is the Kármán number.

*faouzi.laadhari@univ-lyon1.fr 
The universality and values of the dimensionless constants ( $\kappa$, the von Kármán constant and the additive ones $A$ and $B$ ) are the subject of intense discussion (see, e.g., Refs. [4-7]). At the root of this debate are the results obtained at very large Reynolds number in the Princeton "superpipe" facility. First, Zagarola and Smits [8] evaluated $\kappa=0.436 \pm 0.002$, with Pitot tube velocity measurements. By using smaller Pitot tubes, McKeon et al. [9] obtained $\kappa=0.421 \pm 0.002$ in the same facility with appropriate corrections. However, these values remain higher than those commonly obtained in the two other canonical wall flows; namely, the boundary layer and the plane channel [10,11]. Thereafter, Bailey et al. [12], by analyzing five data sets taken in the same facility using Pitot tubes, conventional hot wires, and nanoscale thermal anemometry probes, concluded that the best estimate for the von Kármán constant in this facility can only be obtained with $\pm 5 \%$ accuracy, $\kappa=0.40 \pm 0.02$, due to the inherent measurement uncertainties, thereby requiring more accuracy in the measurement techniques for a better estimate.

These discrepancies in the value of $\kappa$ led some researchers to question its universality and to consider that its value depends on the type of flow [5] or on the Reynolds number [13]. This is in contradiction with the view of supporters of a universal logarithmic law (see, e.g., Marusic et al. [14] among others). The last authors obtained a von Kármán constant $\kappa=0.39$ and $A=4.3$ for boundary layers, pipe flow, and atmospheric surface layers. But it should be noted that, in the laboratory and atmospheric boundary layer datasets they used, the wall shear stress is not measured directly. This is the case of many experimental studies in turbulent boundary layers where the wall shear stress is determined by using empirical correlations, most of which are based on a wall law, such as the Clauser chart [15] with $\kappa=0.41$ and $A=5$, or alternative estimates with different constants [16-18]. Thus, such studies cannot be used unequivocally to provide any conclusion regarding the scaling of the mean velocity profiles in turbulent boundary layer flow [16].

Another point to consider when assessing scaling in wall-bounded flows is the distance over which turbulence evolves before reaching a fully developed flow. In boundary layer flows, this issue is closely linked to the question of the triggering of turbulence [19]. Both numerical [20] and experimental [21-24] works on this issue have shown a significant effect on the development of turbulence downstream of the tripping device and on the strength of the perturbations. In internal flows, the downstream distance after which the flow becomes fully developed is no less than 120 channel widths or pipe diameters $[25,26]$. It is necessary to add to these various issues, which affect the mean velocity profile, the error on the probe position with respect to the wall as well as the position of the center of the channel or pipe and the thickness of the boundary layer. These errors can influence the values of the wall-law constants at very high Reynolds number since, in this situation, a small physical variation is greatly amplified in wall units.

All these issues show that obtaining reliable data at very large Reynolds numbers remains difficult but most of these difficulties can be settled if Reynolds-number effects are correctly accounted for. This is what will be attempted in this paper with results obtained for the logarithmic diagnostic function from highly accurate direct numerical simulations (DNSs) of turbulent plane channel flow. They are extended to other canonical flows by using a similarity variable which makes the mean velocity logarithmic law flow-independent. These results are compared with the finite Reynolds number refinement introduced by Jiménez and Moser [27].

\section{NEW SIMILARITY LAW}

\section{A. Linear behavior of the log-law diagnostic function}

The starting point of this analysis is the observation of the logarithmic diagnostic function $\Xi$, defined so that it is an undeniable indicator of the logarithmic law and of the value of the von Kármán constant $\kappa$, i.e.,

$$
\Xi=\frac{d \ln y^{+}}{d \bar{U}^{+}}=\frac{d \ln \eta}{d \bar{U}^{+}} .
$$



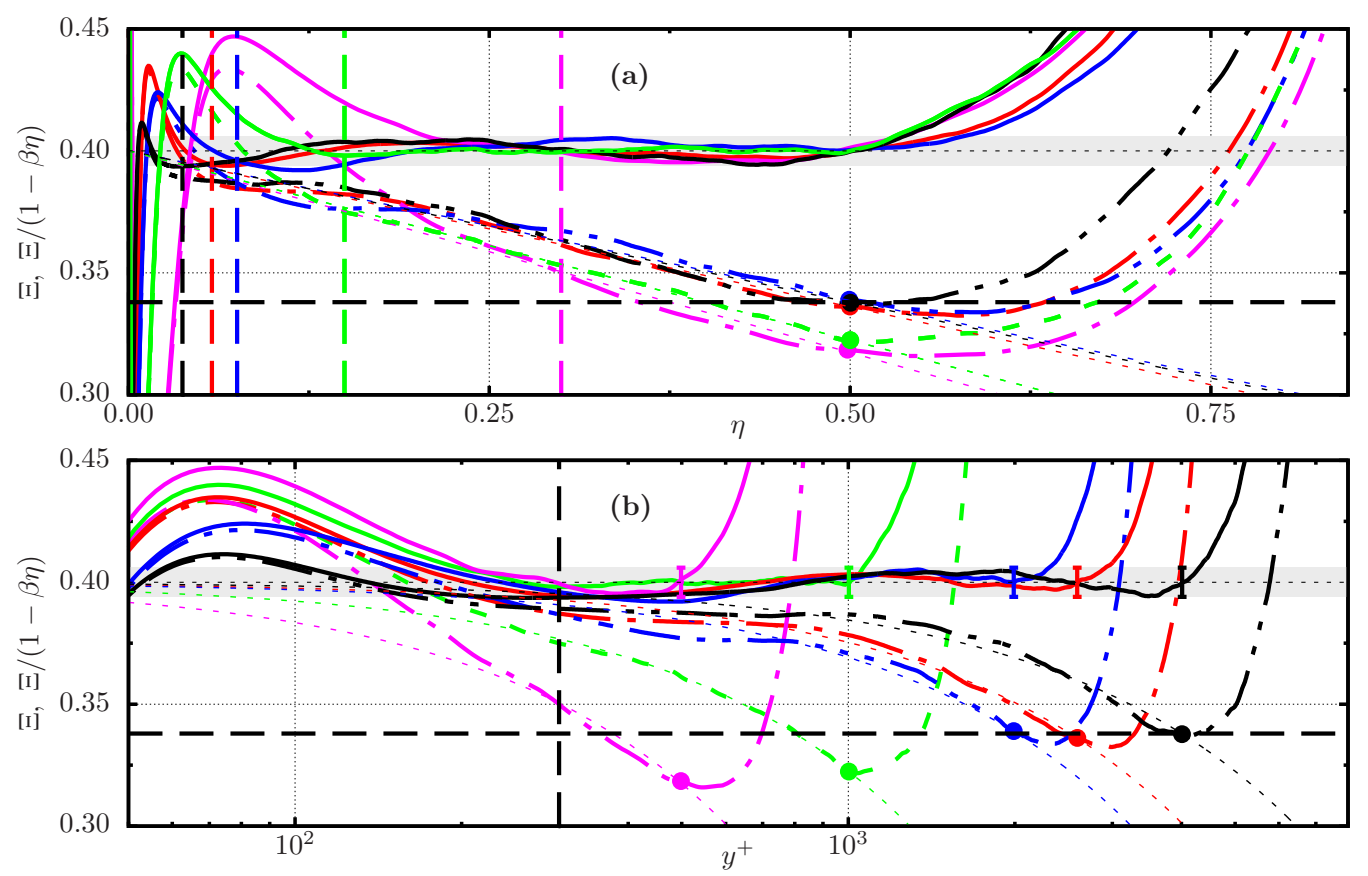

FIG. 1. Log-law diagnostic function $\Xi$ (broken lines) and $\Xi /(1-\beta \eta)$ (solid lines) from DNSs of channel flow. - $----, R_{\tau}=3990,-,---, R_{\tau}=8016$ Yamamoto and Tsuji [28]; -,,$-- R_{\tau}=1000$, ,,$--- R_{\tau}=5186$ Lee and Moser [29]; -,$-- R_{\tau}=2003$ Hoyas and Jiménez [30]. (a) $\Xi$ (broken lines) and $\Xi /(1-\beta \eta)$ (solid lines) as a function of $\eta$. (b) $\Xi$ (broken lines) and $\Xi /(1-\beta \eta)$ (solid lines) plotted against $y^{+}$. Error bars, with an uncertainty of $\pm 1.5 \%$, are located at $y^{+}=\delta^{+} / 2$. Horizontal long dashed line, $\Xi=0.34$. Vertical dashed line, $y^{+}=300$. Bullets denote $\Xi\left(\delta^{+} / 2\right)$. The gray band represents an uncertainty of $\pm 1.5 \%$. The thin dashed lines show Eq. (3).

This function tends to a constant with a value $\kappa$ in the case of the logarithmic laws of Eqs. (1). The profiles of $\Xi$ for various channel flow DNSs are plotted with broken lines as a function of $\eta$ and of $y^{+}$in Figs. 1(a) and 1(b), respectively. The broken lines in Fig. 1(a) clearly highlight the quasilinear decrease with $\eta$, up to $\eta=0.5$, of the diagnostic function with an intercept of 0.4 and a Reynolds-number-dependent slope, denoted $-\kappa \beta$. The profiles of the ratio $\Xi /(1-\beta \eta)$, plotted with solid lines in the two figures, show that the relative departure from the constant value of 0.4 is within $\pm 1.5 \%$. In Fig. 1(a) this ratio exhibits a plateau that extends up to $\eta=0.5$. In Fig. 1(b), where the same quantity is plotted against $y^{+}$, the beginning of the plateau is located at approximately $y^{+}=300$.

Hence, by using the results from available highly reliable DNS data of channel flow at moderately high Reynolds numbers, it is found, as an accurate first approximation, that the logarithmic diagnostic function $\Xi$ decreases linearly with a slope $-\kappa \beta$ and an intercept $\kappa$, i.e.,

$$
\Xi=\frac{1}{y^{+} S^{+}}=\kappa(1-\beta \eta)
$$

with $\kappa=0.4, S^{+}$being the mean velocity gradient, and $\beta$ being a Reynolds-number-dependent variable related to the upper limit of validity of this law, viz.,

$$
\beta=\frac{1}{\eta_{o}}\left(1-\frac{\Xi\left(\eta_{o}\right)}{\kappa}\right)
$$




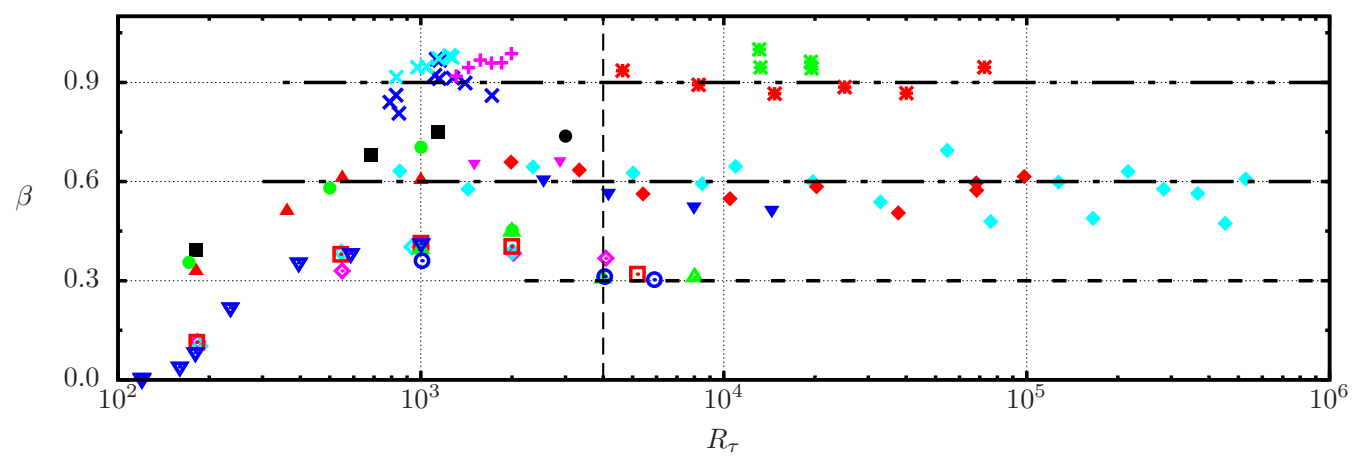

FIG. 2. Evolution with Kármán number of $\beta$ obtained from Eq. (4) with $\eta_{o}=0.5$ for the three canonical flows. Channel flow: $\diamond$, Hoyas and Jiménez [30]; $\nabla$, Laadhari [31]; $\odot$, Schultz and Flack [32]; $\diamond$, Bernardini et al. [33]; $\square$, Lee and Moser [29]; $\triangle$, Yamamoto and Tsuji [28]. Pipe flow: $\diamond$, Zagarola and Smits [8]; $\mathbf{m , ~ W u ~}$

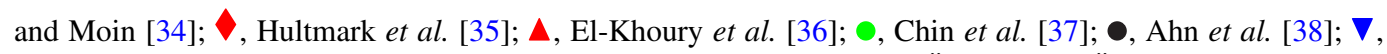
Furuichi et al. [39]; $\boldsymbol{\nabla}$, Bauer et al. [40]. Boundary layer: $\times$, Schlatter and Örlü [20]; $\times$, Örlü and Schlatter [41]; +, Sillero et al. [42]; *, Bailey et al. [43]; *, Vallikivi et al. [44]. - - -, $\beta_{c h}=0.3 ;--, \beta_{p f}=0.6 ;---$, $\beta_{b l}=0.9$. Vertical dashed line: $R_{\tau}=4000$.

The validity of the linear law of Eq. (3) is from $y_{i}^{+}=300$ up to $\eta_{o}=0.5\left(y_{o}^{+}=\delta^{+} / 2\right)$, the lower and upper limits, respectively. It is therefore obvious that this linear law can only be observed if the Kármán number is such that $\delta^{+} \geqslant 600$ and $\left(y_{o}^{+}-y_{i}^{+}\right)$is, at least, of the order of 200 . This is the case for the lowest Kármán number $R_{\tau}=1000$ [29] plotted in Fig. 1 where the normalized diagnostic function is equal to $0.4 \pm 0.004$, with $\beta=0.41$ and within the range $y^{+} \in[300,500]$. It should be noted that the available data do not allow us to verify directly if such linear behavior applies to the canonical turbulent pipe and boundary layer flows since they are mainly restricted to experimental results that produce an inherent large scatter in the diagnostic function profiles due to the mean-velocity gradient evaluation. But, this possibility will be investigated later by using the mean velocity distributions.

These figures show also that the value of $\Xi$ at $\eta=0.5$, highlighted by bullets, reaches a constant value of $\Xi\left(\eta_{o}\right)=0.34$ for $R_{\tau} \geqslant 4000$, leading to a constant value of $\beta=\beta_{c h}=0.3$. This value corresponds to a relative departure of the mean velocity gradient with respect to the value given by $\Xi=\kappa$ of $(\kappa / \Xi-1)$, up to $17 \%$ at the channel quarter-width. This large deviation cannot be the result of errors on the mean velocity gradient, otherwise it would question the validity of all DNS results. Also, the value of $\Xi\left(y_{i}^{+}\right)$, with $y_{i}^{+}=300$, asymptotically approaches the value $\kappa=0.4$ according to the relation

$$
\Xi\left(y_{i}^{+}\right)=\kappa\left(1-\beta_{c h} \frac{y_{i}^{+}}{\delta^{+}}\right)=0.4\left(1-\frac{90}{\delta^{+}}\right)
$$

for $R_{\tau} \geqslant 4000$, thereby leading to a vanishing relative departure from 0.4 with increasing Reynolds number. Hence, if rather a more limited range of distance to the wall is considered, e.g., $y^{+}$within the range $\left[50,0.12 \delta^{+}\right]$, with a negligible variation of $\Xi$, then the value of the von Kármán constant for high Reynolds number is $\kappa=0.4$.

The parameter $\beta$, obtained from Eq. (4) at $\eta_{o}=0.5$, is plotted in Fig. 2 as a function of the Kármán number for the three canonical flows. It is obvious that, after a Reynolds-number-dependent regime, it takes constant but different values for each type of flow. The average values obtained for $R_{\tau} \geqslant 4000$ are $\beta_{c h}=0.3, \beta_{p f}=0.6$, and $\beta_{b l}=0.9$ for channel, pipe, and boundary layer, respectively. These differences in $\beta$ highlight that the geometry affects notably the intensity of 
the mean velocity gradient at $\eta=0.5[37,45,46]$. More particularly, Klewicki [46] evaluated the mean-velocity gradient at the quarter-width of internal wall-bounded turbulent flows and at the half thickness of turbulent boundary layers. He first highlighted the constancy of $d \bar{U}^{+} / d \eta$ at $\eta=0.5$, for sufficiently large Reynolds numbers, and then gave the following limiting values for the three canonical flows: $6.24,7.15$, and 9.86 for channel, pipe, and boundary layer, respectively (see Table 3 page 188 in Ref. [46]). These values are in good agreement with Eq. (3), which yields, for $R_{\tau} \geqslant 4000$, mean velocity gradient values of 5.88, 7.14, and 9.1, with relative departure of $5 \%, 0.1 \%$, and $8 \%$, for channel, pipe, and boundary layer, respectively (for more details, see Appendix A).

Within this context, it is worth mentioning that Jiménez and Moser [27] introduced a refinement of the logarithmic law by using an asymptotic expansion and matching the inner and outer forms of the mean velocity gradient. The correction introduced by these authors with respect to the mean velocity gradient given by the classical logarithmic law is quasiconstant for sufficiently large Reynolds numbers, and flow-dependent, i.e.,

$$
\frac{d \bar{U}^{+}}{d \eta}-\left(\frac{1}{\chi}+\frac{\gamma}{\delta^{+}}\right) \frac{1}{\eta}=\alpha,
$$

while, for the model proposed here, the departure is in addition a function of the distance to the wall, viz.,

$$
\frac{d \bar{U}^{+}}{d \eta}-\frac{1}{\kappa \eta}=\frac{\beta}{\kappa(1-\beta \eta)} .
$$

Then, for sufficiently high Reynolds numbers and by equating the two previous relations, the weighted mean velocity gradient in channel flow at $\eta=0.5$, with $\kappa=\chi=0.4$ and $\beta=0.3$, leads to $\alpha=0.88$ and to

$$
y^{+} \frac{d U^{+}}{d y^{+}}=2.5+0.88 \eta,
$$

which compare very well to Eq. (3), i.e.,

$$
y^{+} \frac{d U^{+}}{d y^{+}}=\frac{2.5}{1-0.3 \eta},
$$

with a relative departure less than $0.7 \%$ in the range $\left[y^{+}=300, \eta=0.5\right]$. It should be noted that Jiménez and Moser [27] also suggested that the value of $\alpha$ for pipe flow is approximately 2.5, which is also well predicted by the present results, since the previous equations for the mean velocity gradient, with $\kappa=0.4, \beta_{p f}=0.6$, and $\eta=0.5$, yield $\alpha=2.14$. A more detailed comparison is presented in Appendix B.

The two refinements suggest that the mean velocity profile never achieves a genuine logarithmic law as claimed by Bernardini et al. [33]. They constitute an alternative to the classical law of the wall and defect law.

\section{B. Logarithmic mean velocity laws}

The integration of Eq. (3) with respect to the wall-normal position, from $y^{+}$or $\eta$ up to $y_{o}^{+}$or $\eta_{o}$, leads to the following logarithmic mean velocity and mean velocity-defect laws

$$
\begin{aligned}
\bar{U}^{+} & =\frac{1}{\kappa} \ln y^{+}-\frac{1}{\kappa} \ln [\kappa(1-\beta \eta)]-\frac{1}{\kappa} \ln a, \\
\bar{U}_{c}^{+}-\bar{U}^{+} & =-\frac{1}{\kappa} \ln \eta+\frac{1}{\kappa} \ln [\kappa(1-\beta \eta)]-\frac{1}{\kappa} \ln b,
\end{aligned}
$$


with $\kappa=0.4$. The range of validity of these expressions extends from $y_{i}^{+}=300$ to $\eta_{o}=0.5$, and $\beta$ is a flow-dependent parameter but invariant for $R_{\tau} \geqslant 4000$. The additional logarithmic term with respect to Eqs. (1), $\ln (1-\beta \eta) / \kappa$, remains relatively weak and varies from $2 \%$ at $\eta=0.2$ for boundary layer flow up to $3.6 \%$ at $\eta=0.5$ for pipe flow, and this for a mean velocity magnitude of $\bar{U}^{+} \simeq 25$.

This term can be interpreted as an anticipation of the mean velocity distribution in the wake region. Indeed, the extension of the upper limit $\eta_{o}$ to $\eta \geqslant 0.2$ leads to a mean velocity and velocitydefect laws more marked by the wake strength, which is stronger in boundary layer flow compared with pipe and channel flows [48-50]. The constants $a$ and $b$ are $a$ priori dependent on the type of confinement and, consequently, the logarithmic friction law

$$
\bar{U}_{c}^{+}\left(R_{\tau}\right)=\frac{1}{\kappa} \ln R_{\tau}-\frac{1}{\kappa} \ln (a b)
$$

will be valid from moderately to infinite Reynolds numbers but depends on the flow geometry through these coefficients.

The empirical constants $a$ and $b$ may be more accurately determined by making the logarithmic laws of Eqs. (5) independent of the parameter $\beta$ using Eq. (3). Interestingly, by replacing $1 /[\kappa(1-$ $\beta \eta)$ ] by the weighted mean velocity gradient $y^{+} S^{+}$, the purely logarithmic mean velocity and mean velocity-defect laws follow

$$
\begin{aligned}
\bar{U}^{+} & =\frac{1}{\kappa} \ln \frac{y^{*}}{a}, \\
\bar{U}_{c}^{+}-\bar{U}^{+} & =-\frac{1}{\kappa} \ln \left(b \eta^{*}\right),
\end{aligned}
$$

with $y^{*}=y^{2} S(y) / v$ and $\eta^{*}=y^{*} / \delta^{+}$. The similarity variable $y^{*}$ is identical to the similarity parameter $\zeta / \kappa^{2}$ of Mellor [51] and to the $Y^{2}$ parameter of McDonald [52] introduced when studying mainstream pressure-gradient effects on the law of the wall. It can be interpreted as the ratio of wall-normal position $y$ to the local shear length scale $v / U_{S}$, with $U_{S}(y)=y S(y)$ being the local shear velocity. Hence, in the classical logarithmic laws of Eq. (1), the wall-friction length scale $v / u_{\tau}$ is used while Eqs. (7) suggest that the length scale changes with the distance to the wall, i.e., $v / U_{S}=v /(y S)$.

It also suggests that the mean shear not only affects turbulent fluctuations but also the mean velocity, in a complex manner that could match the scenario put forward by Jiménez [53]: the mean velocity gradient fixes the rate of production of the velocity fluctuations. If it is increased, the fluctuations are enhanced and create Reynolds shear stress, which in turn modifies the mean velocity profile. In any case, this refers to the close connection between the mean shear and local turbulent processes; thereby giving the possibility to address the finite Reynolds number refinement of the logarithmic law through the dynamical properties of the velocity field and not only by the existence of two length scales, as quoted by Jiménez and Moser [27] regarding the derivation of their refinement.

\section{ASSESSMENT OF THE NEW LOGARITHMIC LAWS}

\section{A. Logarithmic mean velocity law}

The main features of the present analysis will be assessed by using experimental data corresponding, as much as possible, to the largest Reynolds number cases with direct measurement of the wall friction, and available DNS datasets. The choice of only some data used here does not affect the accuracy of the findings. The same conclusions apply to all the pipe-flow results of Örlü et al. [47] and Furuichi et al. [39], and to the boundary layer measurements of Bailey et al. [43] with $R_{\tau} \geqslant 6000$, although it is expected that difficulties will arise because of the evaluation of the mean velocity gradient for experimental data, which introduce a large scatter. 

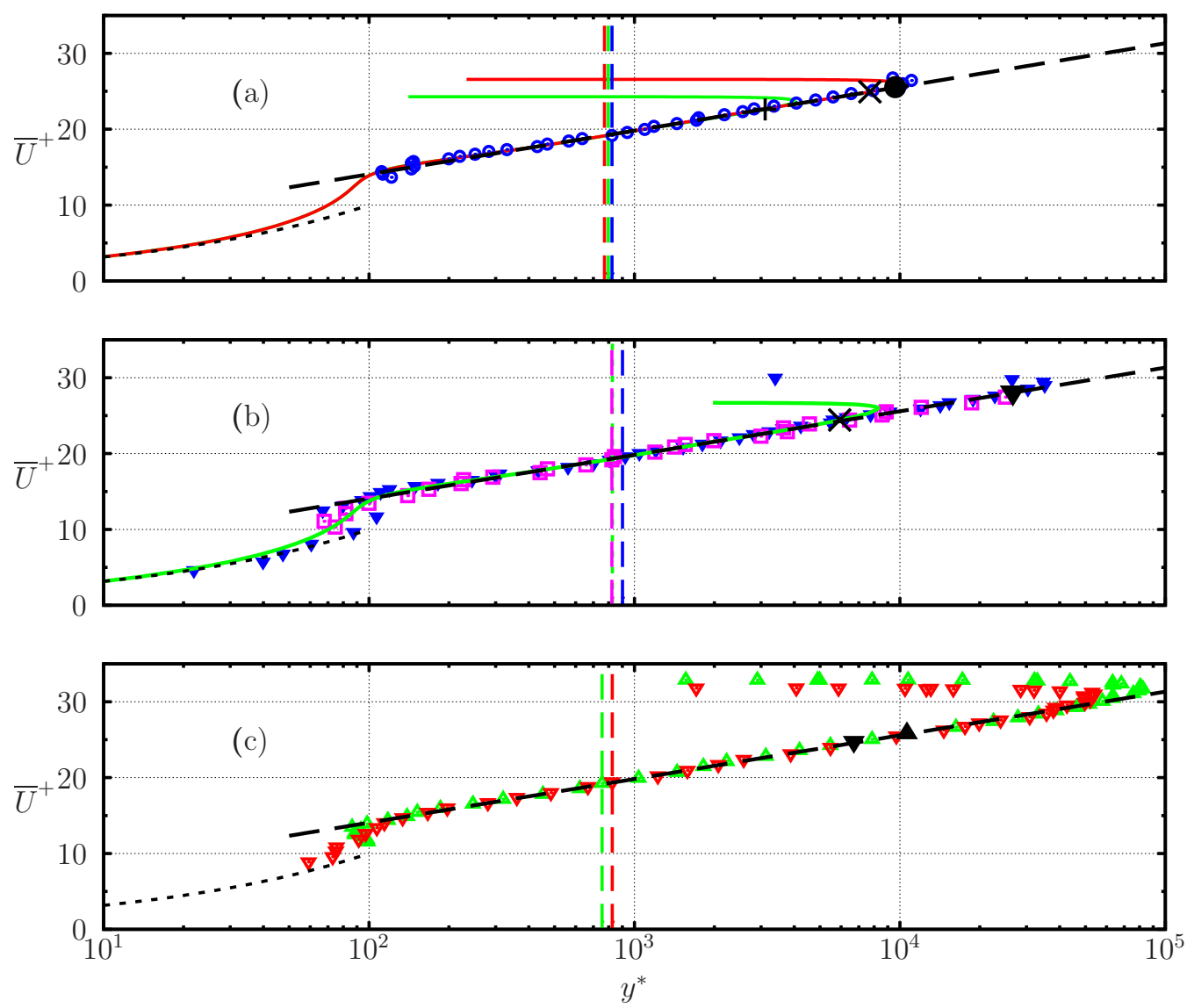

FIG. 3. Mean velocity profiles $\bar{U}^{+}$as a function of $y^{*}$. (a) Channel flow: large black symbols show mean velocity at $\eta=0.5$. (b) Pipe flow: large black symbols show mean velocity at $\eta=0.5$. (c) Boundary layer: large black symbols show mean velocity at $\eta=0.2$. - , Eq. (7a) with $\kappa=0.4$ and $a=0.36 ; \ldots, \sqrt{y^{*}}$. Vertical dashed lines: $y^{+}=300$. All other symbols and lines are defined in Table I.

Figure 3 shows that the logarithmic law of Eq. (7a) with $\kappa=0.4$ and $a=0.36$ is well verified for the three canonical flows in the range $y^{+} \geqslant 300\left(y^{*} \geqslant 750\right.$ and $\left.\bar{U}^{+} \geqslant 19\right)$ and $\eta \leqslant \eta_{o}$, with $\eta_{o}=0.5$ for internal flows and 0.2 for the boundary layer. This lower value of $\eta_{o}$ is due to the presence of small but systematic velocity undershoot just above the logarithmic law observed for boundary layer profiles in Fig. 3(c), which persists with increasing Reynolds number. The values $\kappa$ and $a$ obtained for the data plotted in Fig. 3 and shown in Table I, are in favor of the flow-independent character of the logarithmic law of Eq. (7a) as it takes flow-independent values of 0.4 and 0.36 for $\kappa$ and $a$, respectively, and fits quite well all observations.

Hence, based on the available data, the logarithmic law expressed as a function of the inner and outer normalized wall distance [Eq. (5a)], is found to be different in channel, pipe, and boundary layer flows, only through the presence of the parameter $\beta$, but still unchanged for each flow for $R_{\tau} \geqslant 4000$ and can be interpreted as a limiting law for infinite Reynolds number. However, these results are to be taken with some care since the error on the evaluation of the velocity derivative from experimental profiles can be very large. Therefore, there is no reason to exclude, a priori, a Reynolds number dependence of $\beta$ that can decrease to zero very slowly. The verification of this possibility will for a long time remain beyond reach of current experimental facilities and of high Reynolds number DNSs. 
TABLE I. Estimated values of the logarithmic law constants for the different datasets used.

\begin{tabular}{|c|c|c|c|c|c|c|c|}
\hline Authors & $\begin{array}{c}\text { Type } \\
\text { of flow }\end{array}$ & $R_{\tau}$ & $\kappa$ & $\begin{array}{c}\beta^{\mathrm{b}} \\
{[\text { Eq. (4)] }}\end{array}$ & $a$ & $b$ & $\begin{array}{l}\text { Symbols } \\
\text { and lines }\end{array}$ \\
\hline Hoyas and Jiménez [30] & DNS-CH & 2003 & 0.4 & 0.388 & 0.363 & 0.33 & - \\
\hline Yamamoto and Tsuji [28] & DNS-CH & 8016 & 0.403 & 0.31 & 0.361 & 0.36 & - \\
\hline Yamamoto and Tsuji [28] & DNS-CH & 3986 & 0.403 & 0.307 & 0.355 & 0.35 & - \\
\hline Bernardini et al. [33] & DNS-CH & 4079 & 0.4 & 0.368 & 0.376 & 0.34 & \\
\hline Lee and Moser [29] & DNS-CH & 5186 & 0.401 & 0.32 & 0.35 & 0.348 & \\
\hline Schultz and Flack [32] & Exp. $\mathrm{CH}$ & 5895 & 0.395 & 0.303 & 0.379 & 0.367 & $\odot$ \\
\hline Bailey et al. [43] & Exp. BL & 13100 & 0.402 & $1.0(0.78)$ & 0.338 & 0.107 & $\nabla$ \\
\hline Bailey et al. [43] & Exp. BL & 19518 & 0.405 & $0.94(0.77)$ & 0.322 & 0.103 & $\triangle$ \\
\hline Ahn et al. [38] & DNS-PF & 3008 & 0.4 & 0.73 & 0.36 & 0.2 & $\bar{\sigma}$ \\
\hline Örlü et al. [47] & Exp. PF & 32000 & 0.396 & $\mathrm{c}$ & 0.39 & 0.32 & $\bullet$ \\
\hline Furuichi et al. [39] & Exp. PF & 14400 & 0.403 & 0.56 & 0.325 & 0.263 & $\nabla$ \\
\hline
\end{tabular}

${ }^{\mathrm{a}} \mathrm{CH}$ : channel, PF: pipe, BL: boundary layer flows.

${ }^{\mathrm{b}}$ Values obtained with $\eta_{o}=0.5$, while those in brackets are obtained with $\eta_{o}=0.2$.

${ }^{\mathrm{c}}$ Unavailable since data are limited to $\eta \leqslant 0.22$.

\section{B. Logarithmic mean velocity-defect law}

In Fig. 4, the mean velocity deficit is plotted as a function of $\eta^{*}$ for the three flows. As expected, the slope is flow-independent, $1 / \kappa=2.5$, while the coefficient $b$ in Eq. (7b) is different for each flow. The fitting of the data in the range $300 / \delta^{+} \leqslant \eta \leqslant \eta_{o}$ with respect to this relation gives the values of the coefficient $b_{c h}=0.36, b_{p f}=0.25$, and $b_{b l}=0.11$ for channel, pipe, and boundary layer flow, respectively. The channel-flow measurements of Schultz and Flack [32], well described by the fitting coefficients $\kappa=0.396 \pm 0.004$ and $b=0.366 \pm 0.005$, are in good agreement with the DNS profile at $R_{\tau}=5186$ [29]. Note that the value of $b=0.2$ (see Table I) obtained for the DNS defect law profile of Ahn et al. [38] is less than 0.25 since it is at a Kármán number less than 4000, where low Reynolds number effects are present on $b$. In the case of the boundary layer mean velocity-defect profiles of Fig. 4(c), the undershoot observed in Fig. 3(c) appears therefore as an overshoot, and the departure from the logarithmic law is observed beyond the position $\eta=0.2$ $\left(\eta^{*} \approx 0.55\right)$, highlighted by the large black symbols.

\section{LOGARITHMIC FRICTION LAWS}

With the previous results it is now possible to specify the friction law for the most important two flows in engineering application, namely the pipe flow and the zero-pressure-gradient turbulent boundary layer. Note that for evaluating the power-loss in internal flows (the mean streamwise pressure gradient by the mean flow rate) the bulk velocity $\bar{U}_{b}$ is needed. The classical approach for obtaining it is from the centerline velocity by assuming a constant value to the Zagarola-Smits [8] velocity scale $\xi=\bar{U}_{c}^{+}-\bar{U}_{b}^{+}$(see Schlichting Ref. [54], p. 609). The value of $\xi=4.3$, for pipe flow is obtained from the average value of 4.34 given by Zagarola and Smits (Ref. [55], Fig. 22), 4.28 by McKeon and Morrison [Ref. [56], Fig. 2(b)], and 4.26 by Furuichi et al. (Ref. [39], Fig. 12).

Then, for the pipe flow with $\kappa=0.4, a=0.36, b_{p f}=0.25$, and $\xi_{p f}=4.3$, the universal law of friction for a smooth pipe proposed by Schlichting (Ref. [54], page 610) is recovered. First, as a function of Kármán number [see Schlichting Ref. [54], relation (20.29)],

$$
\bar{U}_{b}^{+}=2.5 \ln R_{\tau}+1.72
$$



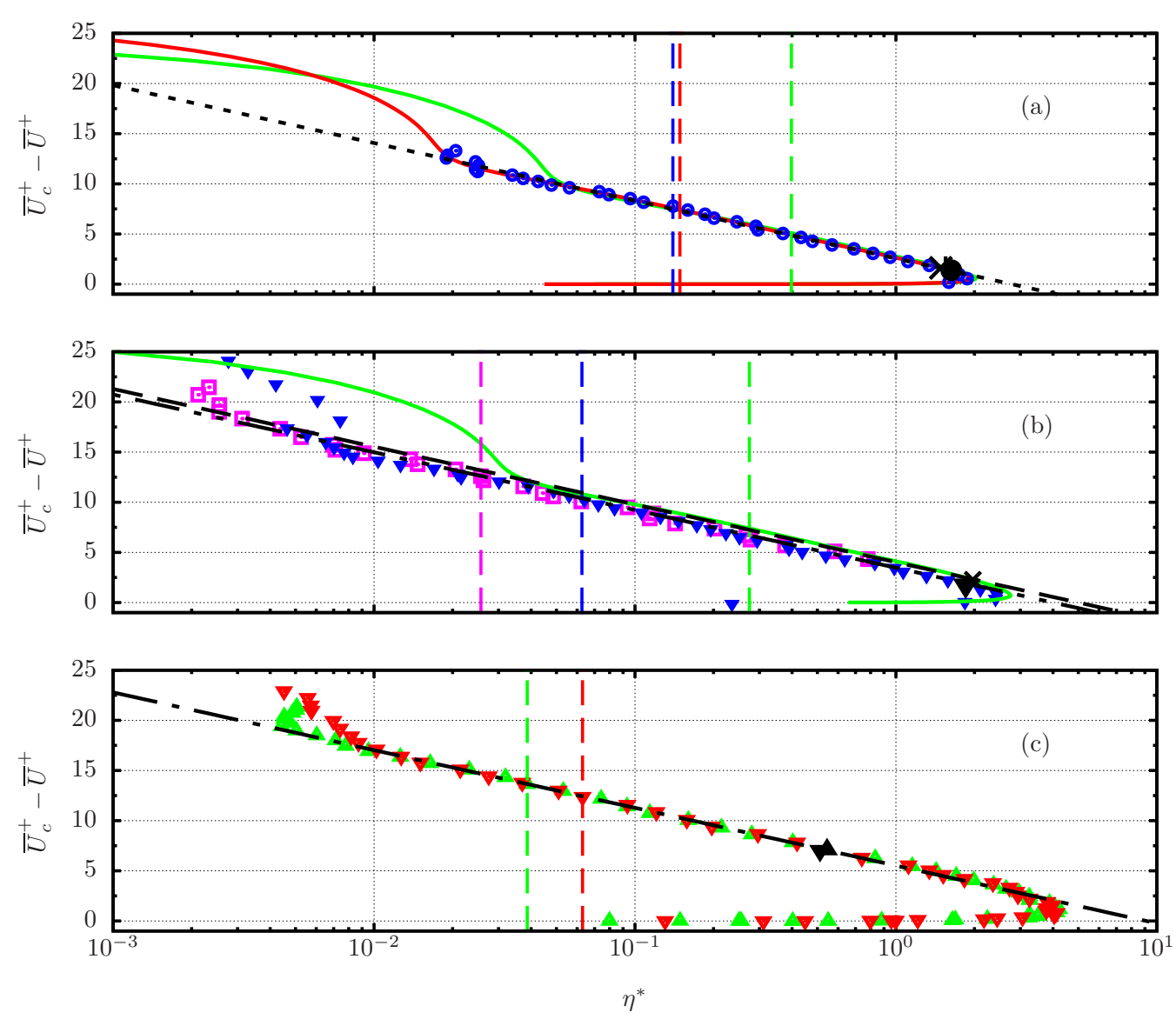

FIG. 4. Mean velocity-defect profiles as a function of $\eta^{*}$. (a) Channel flow: dotted line, Eq. (7b) with $\kappa=0.4$ and $b=0.36$; large black symbols, mean velocity deficit at $\eta_{o}=0.5$. (b) Pipe flow: dashed line, Eq. (7b) with $\kappa=0.40$ and $b=0.2$; dashed-double dotted line, Eq. (7b) with $\kappa=0.4$ and $b=0.25$; large black symbols, mean velocity deficit at $\eta_{o}=0.5$. (c) Boundary layer: dashed-dotted line, Eq. (7b) with $\kappa=0.4$ and $b=0.11$; large black symbols, mean velocity deficit at $\eta_{o}=0.2$. Vertical dashed lines: $y^{+}=300$. All other symbols and lines are defined in Table I.

and second, for the Darcy-Weisbach friction factor, $\lambda=8 / \bar{U}_{b}^{+2}$, as a function of the bulk Reynolds number, $R_{b}=\bar{U}_{b} D / \nu$ :

$$
\frac{1}{\sqrt{\lambda}}=2.035 \log _{10}\left(R_{b} \sqrt{\lambda}\right)-0.92
$$

Note that $\lambda$ can be explicitly expressed as a function of the bulk Reynolds number by using the principal branch of the Lambert $W_{0}$ function (see, e.g., More, Ref. [57])

$$
\lambda=8 \kappa^{2}\left[W_{0}\left(\frac{\kappa R_{b}}{2 a b_{p f} \exp (\kappa \xi)}\right)\right]^{-2} \simeq \frac{1.28}{\left[W_{0}\left(0.4 R_{b}\right)\right]^{2}} .
$$

Finally, for the turbulent boundary layer, the friction law defined by the triplet of parameters $\kappa=0.4, a=0.36, b_{b l}=0.11$ in Eq. (6) leads to

$$
\bar{U}_{\infty}^{+}=2.5 \ln R_{\tau}+8.07
$$


Recently, Heinz (Ref. [58], Fig. A.2d) provides a relation between the momentum thickness Reynolds number $R_{\theta}$ and the Kármán number, i.e., $R_{\tau}=0.31 R_{\theta}$, which leads to the skin friction relation of the Coles-Fernholz type:

$$
C_{f}=\frac{2}{\left[2.5 \ln R_{\theta}+5.1\right]^{2}}
$$

This friction law lies between the commonly used version, utilizing $\kappa=0.41$ and an additive constant $C=5$, and the one proposed by Nagib et al. [59] with $\kappa=0.384$ and $C=4.127$.

\section{CONCLUSION}

The most important result obtained in this study is that, by replacing the wall shear velocity by the local shear velocity for scaling the wall distance, strong evidence appears for the existence of a flow-independent logarithmic law for canonical turbulent wall-bounded flows [Eq. (7a)], with a validity range from $y^{+}=300$ up to $\eta=0.5$ for pipe and channel flows and $\eta=0.2$ for boundary layer flow. The slope of this purely logarithmic law is the inverse of the von Kármán constant with unambiguously $\kappa=0.4$ for the three canonical flows.

On the other hand, when the normalized mean velocity is expressed against the classical variables $y^{+}$and $\eta$, then it is flow-dependent through the presence of the parameter $\beta$ in Eq. (5a). This parameter reflects the interaction of the outer region with the near-wall region; and its constancy for $R_{\tau} \geqslant 4000$ implies that the influence of the outer length scale is present permanently. The improvement of the present laws through the term $1-\beta \eta$ leads to a more precise determination of the empirical constants over a greater range of wall distance. In addition, the velocity defect law [Eq. (7b)] is shifted by a flow-dependent constant function of $b$.

Also, by relating the Zagarola-Smits velocity scale and the momentum-thickness Reynolds number to the evolution of the mean centerline velocity of Eq. (6), the classical friction laws for turbulent pipe and zero-pressure-gradient boundary layer flows were obtained with a single von Kármán constant $\kappa=0.4$.

It should be noted that the present model for the weighted mean velocity gradient compares very well with the model introduced by Jiménez and Moser [27]. However, unlike the model proposed in Ref. [27], the present model is directly related to a simple alternative expression for the log-law, Eq. (7a), function of a local shear-based wall distance. One of its features is that the mean wall friction velocity appears only on the left-hand side, so that it can be obtained directly by accurate measurements of the mean streamwise velocity and its wall-normal gradient, relatively far from the wall, up to $\eta=0.2$.

This suggests an alternative to the classical empirical method for assessing the wall friction in canonical wall bounded flows and more particularly in the case of zero-pressure-gradient turbulent boundary layers. More precisely, in this case the wall friction velocity is given by $u_{\tau}=\Xi y S$, so that the relative departure from the value given by the classical log-law, i.e., $\kappa y S$, is $(1-\kappa / \Xi)=$ $-\beta_{b l} \eta /\left(1-\beta_{b l} \eta\right)$ and varies from $-1 \%$ at $\eta=0.01$ to $-22 \%$ at $\eta=0.2$.

\section{ACKNOWLEDGMENTS}

I thankfully acknowledge all the colleagues who made their data available to the community or who provided me with their valuable data. I am very thankful to my colleague Dr. Wouter J. T. Bos for his encouragement and helpful comments throughout this study. This work was granted access to the HPC ressources of the FLMSN, "Fédération Lyonnaise de Modélisation et Sciences Numériques," partner of EQUIPEX EQUIP@MESO. 
TABLE II. Mean velocity gradient $d \bar{U}^{+} / d \eta$ at $\eta=0.5$.

\begin{tabular}{lccc}
\hline \hline Flow type & Klewicki (Ref. [46]) & Present Eq. (3) & RD (\%) \\
\hline Channel & 6.24 & 5.88 & 6 \\
Pipe & 7.15 & 7.14 & 0.1 \\
Boundary layer & 9.86 & 9.09 & 8 \\
\hline \hline
\end{tabular}

\section{APPENDIX A: COMPARISON WITH KLEWICKI (REF. [46]) ESTIMATIONS OF MEAN VELOCITY GRADIENT AND VELOCITY DEFICIT AT $\boldsymbol{\eta}=\mathbf{0 . 5}$}

Klewicki [46] has pointed out the differences in the mean-velocity distribution at the quarterwidth of internal wall-bounded turbulent flows and the half-thickness of the turbulent boundary layer. He gives (see Ref. [46], Table 3, page 188) the values of the mean velocity gradient and the mean velocity deficit at $\eta=0.5$ for the three canonical flows. These values are well predicted by the relations obtained here and more particularly for internal flows, bearing in mind that in the case of boundary layer flow, the present logarithmic laws of Eq. (5) are valid only up to $\eta=0.2$.

In Tables II and III the values given by this author are compared with those given by the laws obtained in the present work for the mean velocity gradient and the mean velocity deficit at $\eta=0.5$ with the corresponding relative departure (RD).

\section{APPENDIX B: COMPARISON WITH JIMÉNEZ AND MOSER MODEL (REF. [27], SECTION 4A)}

The refinement developed by Jiménez and Moser [27] corresponds to the introduction of two supplementary terms in the expression of the weighted mean velocity gradient (see relation 4.9 in Ref. [27]) with respect to the constant value $1 / \chi$ of the classical law (i) a high Reynolds-number vanishing term and (ii) a Reynolds-number-dependent linear term, i.e.,

$$
\frac{1}{\Xi}=y^{+} \frac{d \bar{U}^{+}}{d y^{+}}=\frac{1}{\chi}+\frac{\gamma}{\delta^{+}}+\alpha \eta,
$$

within the range of validity from $y^{+}=350$ up to $\eta=0.45$, as found by these authors.

This model can be recovered from the present linear diagnostic function of Eq. (3), when expressed with a first-order expansion around $\eta_{\emptyset}$, i.e.,

$$
\frac{1}{\Xi}=\frac{1}{\kappa(1-\beta \eta)} \approx \frac{1-2 \beta \eta_{\emptyset}}{\kappa\left(1-\beta \eta_{\emptyset}\right)^{2}}+\frac{\beta}{\kappa\left(1-\beta \eta_{\emptyset}\right)^{2}} \eta,
$$

with $\eta_{\emptyset}$ within this range.

TABLE III. Mean velocity deficit $\bar{U}_{c}^{+}-\bar{U}^{+}$at $\eta=0.5$.

\begin{tabular}{lccr}
\hline \hline Flow type & Klewicki (Ref. [46]) & Present Eq. (5b) & RD (\%) \\
\hline Channel & 1.75 & 1.59 & 9.6 \\
Pipe & 2.03 & 2.02 & 0.5 \\
Boundary layer & 2.66 & 3.47 & 26.4 \\
\hline \hline
\end{tabular}


TABLE IV. Values of parameters in Eqs. (B1) and (B2) from different DNS of turbulent channel flow.

\begin{tabular}{lcccccccc}
\hline \hline Authors & $R_{\tau}$ & $\beta^{\mathrm{a}}$ & $\chi$ & $\gamma$ & {$\left[\frac{1}{\chi}+\frac{\gamma}{\delta^{+}}\right]$} & {$\left[\frac{1-2 \beta \eta_{\varnothing}}{\kappa\left(1-\beta \eta_{\S}\right)^{2}}\right]^{\mathrm{b}}$} & $\alpha$ & {$\left[\frac{\beta}{\kappa\left(1-\beta \eta_{\varnothing}\right)^{2}}\right]^{\mathrm{b}}$} \\
\hline Jiménez and Moser $^{\mathrm{c}}$ & 2003 & 0.388 & 0.402 & 150 & 2.56 & 2.47 & 1 & 1.2 \\
${\text { Bernardini } \text { et al. }{ }^{\mathrm{d}}}^{4}$ & 4079 & 0.37 & 0.41 & 180 & 2.48 & 2.47 & 1.15 & 1.13 \\
Lee and Moser $^{\mathrm{c}}$ & 5186 & 0.32 & & & $2.586(2.49)^{\mathrm{f}}$ & 2.48 & $0.2(0.94)^{\mathrm{f}}$ & 0.95 \\
Present prediction & $\geqslant 4000$ & 0.3 & & & & 2.48 & & 0.88 \\
\hline \hline
\end{tabular}

$\overline{\mathrm{a}} \beta$ from Eq. (4).

bobtained with $\kappa=0.4$ and $\eta_{\emptyset}=0.26$.

${ }^{\mathrm{c}}$ References [27,30].

${ }^{\mathrm{d}}$ Reference [33].

${ }^{\mathrm{e}}$ Reference [29].

${ }^{\mathrm{f}}$ Values in brackets are obtained by fitting the data of Ref. [29] in the range $y^{+}=350, \eta=0.45$.

Then, equating terms of the same order on right-hand sides of Eqs. (B1) and (B2) leads to the following relations:

$$
\begin{aligned}
\frac{1}{\chi}+\frac{\gamma}{\delta^{+}} & =\frac{1-2 \beta \eta_{\emptyset}}{\kappa\left(1-\beta \eta_{\emptyset}\right)^{2}}, \\
\alpha & =\frac{\beta}{\kappa\left(1-\beta \eta_{\emptyset}\right)^{2}} .
\end{aligned}
$$

Note that another relation exists between $\alpha$ and $\beta$ and is obtained by equating the two expressions of $\Xi$ provided by Eqs. (B1) and (3) at the position $\eta_{o}$, whence follows, with $\delta^{+} \rightarrow+\infty$ and $\kappa=\chi$,

$$
\alpha=\frac{\beta}{\kappa\left(1-\beta \eta_{o}\right)}
$$

which, with Eq. (B3b), yields

$$
\eta_{\emptyset}=\frac{1-\sqrt{1-\beta \eta_{o}}}{\beta},
$$

and to the value $\eta_{\emptyset}=0.26$ (with $\beta=0.3$ and $\eta_{o}=0.5$ for channel flow), approximately the middle of the range where $\Xi$ is linear.

The left- and right-hand sides of Eqs. (B3) are compared in Table IV for three channel flow DNS results and the agreement between them is very good for the results of Jiménez and Moser [27] and Bernardini et al. [33]. The value $\alpha=0.2$ of Lee and Moser [29] in Table IV is very low compared with the others results. It seems to have been obtained with a data fitting in the range $y^{+}=350$ to $\eta=0.16\left[y^{+}=830\right.$ in their Fig. 3(c)], smaller than the range used by Jiménez and Moser [27] and Bernardini et al. [33]. The fitting of their data at $R_{\tau}=5186$ in the range $\left[y^{+}=350, \eta=0.45\right.$ ] yields the set of values $1 / \chi+\gamma / \delta^{+}=2.49$ and $\alpha=0.94$ (enclosed in parentheses in Table IV) that compare very well with the present predictions of 2.48 and 0.95 , respectively.

[1] T. von Kármán, Mechanical similitude and turbulence, Technical Memorandum 611, NACA (1931).

[2] L. Prandtl, Zur Turbulenten Strömung in Rohren und längs Platten, in Ergebnisse der Aerodynamischen Versuchsanstalt zu Göttingen (Universitätsverlag Göttingen, 1932), pp. 18-29.

[3] T. von Kármán, Turbulence and skin friction, J. Aeronaut. Sci. 1, 1 (1934). 
[4] M. H. Buschmann and M. Gad-el Hak, Recent developments in scaling of wall-bounded flows, Prog. Aerosp. Sci. 42, 419 (2006).

[5] H. M. Nagib and K. A. Chauhan, Variations of von Kármán coefficient in canonical flows, Phys. Fluids 20, 101518 (2008).

[6] J. C. Klewicki, Reynolds number dependence, scaling, and dynamics of turbulent boundary layers, J. Fluids Eng. 132, 094001 (2010).

[7] A. J. Smits, B. J. McKeon, and I. Marusic, High-Reynolds number wall turbulence, Annu. Rev. Fluid Mech. 43, 353 (2011).

[8] M. V. Zagarola and A. J. Smits, Scaling of the Mean Velocity Profile for Turbulent Pipe Flow, Phys. Rev. Lett. 78, 239 (1997).

[9] B. J. McKeon, J. Li, W. Jiang, J. F. Morrison, and A. J. Smits, Further observations on the mean velocity distribution in fully developed pipe flow, J. Fluid Mech. 501, 135 (2004).

[10] J. M. Österlund, A. V. Johansson, H. M. Nagib, and M. H. Hites, A note on the overlap region in turbulent boundary layers, Phys. Fluids 12, 1 (2000).

[11] E.-S. Zanoun, F. Durst, and H. Nagib, Evaluating the law of the wall in two-dimensional fully developed turbulent channel flows, Phys. Fluids 15, 3079 (2003).

[12] S. C. C. Bailey, M. Vallikivi, M. Hultmark, and A. J. Smits, Estimating the value of von Kármán's constant in turbulent pipe flow, J. Fluid Mech. 749, 79 (2014).

[13] M. Wosnik, L. Castillo, and W. K. George, A theory for turbulent pipe and channel flows, J. Fluid Mech. 421, 115 (2000).

[14] I. Marusic, J. P. Monty, M. Hultmark, and A. J. Smits, On the logarithmic region in wall turbulence, J. Fluid Mech. 716, R3 (2013).

[15] F. H. Clauser, The Turbulent Boundary Layer (Elsevier, 1956), pp. 1-51.

[16] D. B. De Graaff and J. K. Eaton, Reynolds-number scaling of the flat-plate turbulent boundary layer, J. Fluid Mech. 422, 319 (2000).

[17] N. Hutchins, T. B. Nickels, I. Marusic, and M. S. Chong, Hot-wire spatial resolution issues in wallbounded turbulence, J. Fluid Mech. 635, 103 (2009).

[18] D. T. Squire, C. Morrill-Winter, N. Hutchins, M. P. Schultz, J. C. Klewicki, and I. Marusic, Comparison of turbulent boundary layers over smooth and rough surfaces up to high Reynolds numbers, J. Fluid Mech. 795, 210 (2016).

[19] N. Hutchins, Caution: Tripping hazards, J. Fluid Mech. 710, 1 (2012).

[20] P. Schlatter and R. Örlü, Assessment of direct numerical simulation data of turbulent boundary layers, J. Fluid Mech. 659, 116 (2010).

[21] L. P. Erm and P. N. Joubert, Low-Reynolds-number turbulent boundary layers, J. Fluid Mech. 230, 1 (1991).

[22] L. Castillo and T. G. Johansson, The effects of the upstream conditions on a low Reynolds number turbulent boundary layer with zero pressure gradient, J. Turbul. 3, N31 (2002).

[23] T. Johansson and R. Karlsson, Measurement issues in high Reynolds number flows, in 40th AIAA Aerospace Sciences Meeting \& Exhibit (American Institute of Aeronautics and Astronautics, Reno, NV, USA, 2002).

[24] I. Marusic, K. A. Chauhan, V. Kulandaivelu, and N. Hutchins, Evolution of zero-pressure-gradient boundary layers from different tripping conditions, J. Fluid Mech. 783, 379 (2015).

[25] J. P. Monty, Developments in smooth wall turbulent duct flows, Ph.D. thesis, University of Melbourne, Department of Mechanical and Manufacturing Engineering, 2005.

[26] E.-S. Zanoun, E. Öngüner, and C. Egbers, Conventional measuring probes in the wall layer of turbulent subsonic ducted flows, Thermophys. Aeromechanics 23, 329 (2016).

[27] J. Jiménez and R. D. Moser, What are we learning from simulating wall turbulence? Philos. Trans. R. Soc., A 365, 715 (2007).

[28] Y. Yamamoto and Y. Tsuji, Numerical evidence of logarithmic regions in channel flow at $\operatorname{Re}_{\tau}=8000$, Phys. Rev. Fluids 3, 012602(R) (2018).

[29] M. Lee and R. D. Moser, Direct numerical simulation of turbulent channel flow up to $R e_{\tau} \approx 5200$, J. Fluid Mech. 774, 395 (2015). 
[30] S. Hoyas and J. Jiménez, Scaling of the velocity fluctuations in turbulent channels up to $R e_{\tau}=2003$, Phys. Fluids 18, 011702 (2006).

[31] F. Laadhari, Reynolds number effect on the dissipation function in wall-bounded flows, Phys. Fluids 19, 038101 (2007).

[32] M. P. Schultz and K. A. Flack, Reynolds-number scaling of turbulent channel flow, Phys. Fluids 25, 025104 (2013).

[33] M. Bernardini, S. Pirozzoli, and P. Orlandi, Velocity statistics in turbulent channel flow up $R e_{\tau}=4000$, J. Fluid Mech. 742, 171 (2014).

[34] X. Wu and P. Moin, A direct numerical simulation study on the mean velocity characteristics in turbulent pipe flow, J. Fluid Mech. 608, 81 (2008).

[35] M. Hultmark, M. Vallikivi, S. C. C. Bailey, and A. J. Smits, Turbulent Pipe Flow at Extreme Reynolds Numbers, Phys. Rev. Lett. 108, 094501 (2012).

[36] G. K. El-Khoury, P. Schlatter, A. Noorani, P. F. Fischer, G. Brethouwer, and A. V. Johansson, Direct numerical simulation of turbulent pipe flow at moderately high Reynolds numbers, Flow, Turbul. Combust. 91, 475 (2013).

[37] C. Chin, J. Monty, and A. Ooi, Reynolds number effects in DNS of pipe flow and comparison with channels and boundary layer, Int. J. Heat Fluid Flow 45, 33 (2014).

[38] J. Ahn, J. H. Lee, J. Lee, J. Kang, and J. J. Sung, Direct numerical simulation of a 30R long turbulent pipe flow at $\operatorname{Re}_{\tau}=3008$, Phys. Fluids 27, 065110 (2015).

[39] N. Furuichi, Y. Terao, Y. Wada, and Y. Tsuji, Friction factor and mean velocity profile for pipe flow at high Reynolds numbers, Phys. Fluids 27, 095108 (2015).

[40] C. Bauer, D. Feldmann, and C. Wagner, On the convergence and scaling of high-order statistical moments in turbulent pipe flow using direct numerical simulations, Phys. Fluids 29, 125105 (2017).

[41] R. Örlü and P. Schlatter, Comparison of experiments and simulations for zero pressure gradient turbulent boundary layers at moderate Reynolds numbers, Exp. Fluids 54, 1547 (2013).

[42] J. A. Sillero, J. Jiménez, and R. D. Moser, One-point statistics for turbulent wall-bounded flows at Reynolds numbers up to $\delta^{+} \approx 2000$, Phys. Fluids 25, 105102 (2013).

[43] S. C. C. Bailey, M. Hultmark, J. P. Monty, P. H. Alfredsson, M. S. Chong, R. D. Duncan, J. H. M. Fransson, N. Hutchins, I. Marusic, B. J. McKeon, H. M. Nagib, R. Örlü, A. Segalini, A. J. Smits, and R. Vinuesa, Obtaining accurate mean velocity measurements in high Reynolds number turbulent boundary layers using Pitot tubes, J. Fluid Mech. 715, 642 (2013).

[44] M. Vallikivi, M. Hultmark, and A. J. Smits, Turbulent boundary layer statistics at very high Reynolds number, J. Fluid Mech. 779, 371 (2015).

[45] F. Laadhari, An apparent symmetry property of the mean velocity gradient in turbulent Poiseuille flows and its implications, Phys. Fluids 23, 101705 (2011).

[46] J. C. Klewicki, A turbulent wall-flow vorticity consistent with mean dynamics, J. Fluid Mech. 737, 176 (2013). Supplemental Material https://doi.org/10.1017/jfm.2013.565.

[47] R. Örlü, T. Fiorini, A. Segalini, G. Bellani, A. Talamelli, and P. H. Alfredsson, Reynolds stress scaling in pipe flow turbulence-first results from CICLoPE, Philos. Trans. R. Soc. London, Ser. A 375, 20160187 (2017).

[48] R. L. Panton, Review of wall turbulence as described by composite expansions, Appl. Mech. Rev. 58, 1 (2005).

[49] J. P. Monty, N. Hutchins, H. C. H. Ng, I. Marusic, and M. S. Chong, A comparison of turbulent pipe, channel and boundary layer flows, J. Fluid Mech. 632, 431 (2009).

[50] J. Jiménez, S. Hoyas, P. P. Simens, and Y. Mizuno, Turbulent boundary layers and channels at moderate Reynolds numbers, J. Fluid Mech. 657, 335 (2010).

[51] G. L. Mellor, The effects of pressure gradients on turbulent flow near a smooth wall, J. Fluid Mech. 24, 255 (1966).

[52] H. McDonald, The effect of pressure gradient on the law of the wall in turbulent flow, J. Fluid Mech. 35, 311 (1969).

[53] J. Jiménez, Wall turbulence with arbitrary mean velocity profiles, in Center for Turbulence Research Annual Research Briefs (Stanford University, Stanford, CA, 2010). 
[54] H. Schlichting, Boundary-Layer Theory, 7th ed. (McGraw-Hill, New York, 1979).

[55] M. V. Zagarola and A. J. Smits, Mean-flow scaling of turbulent pipe flow, J. Fluid Mech. 373, 33 (1998).

[56] B. J. McKeon and J. F. Morrison, Asymptotic scaling in turbulent pipe flow, Philos. Trans. R. Soc., A 365, 771 (2007).

[57] A. A. More, Analytical solutions for the Colebrook and White equation and for pressure drop in ideal gas flow in pipes, Chem. Eng. Sci. 61, 5515 (2006).

[58] S. Heinz, On mean flow universality of turbulent wall flows. I. High Reynolds number flow analysis, J. Turbul. 19, 929 (2018).

[59] H. M. Nagib, K. A. Chauhan, and P. A. Monkewitz, Approach to an asymptotic state for zero pressure gradient turbulent boundary layers, Philos. Trans. R. Soc., A 365, 755 (2007). 